\title{
How Do We Work? Metacognition in Creative and Collaborative Practices
}

\section{Byron Wolfe}

Temple University

Seher Erdogan Ford

Temple U niversity
Why teach collaboration? Considering the context of growing emphasis on collaborative work in arts and design education, what it actually entails and how it works warrant a closer look. Institutions are motivated to help students launch multidisciplinary creative careers, while educators assign collaborative projects for students and engage in similar activities in their own practices. Given this momentum, a re existing pedagogical models in creative disciplines designed appropriately to foster healthy and effective collaborations? M oreover, is there sufficient common understanding or language for what might constitute best practices for initiating and maintaining sustainable collaborations?

These questions arise regularly within the context of our institution, Tyler School of Art and A rchitecture, which is part of Temple University in Philadelphia, Pennsylvania. The school includes the departments of Architecture and Environmental Design, Art Education and Community Arts Practices, Art History, Studio Art, and Graphic and Interactive Design. It recently updated its structure and adopted a $n$ ame that c aptures its breadth of p rograms to support cross-disciplinary s tudy and reflect current understanding of creative practice and research.

One of us being a professor in Studio Art with a background in Photography and the other in Architecture and Environmental Design, our collective experience and shared interests in interdisciplinary engagements motivated us to design and co-teach a new, graduate-level course focusing on collaboration and the creative process. Following preparations and planning for about a year, we taught the course titled "Collaboration and Creativity" three times since its first iteration in the fall of 2017. Each semester varied widely in terms of the number of students enrolled, background and expectations both on the part of the students as well as us, as instructors. So far the cohort has included students from architecture, photography, ceramics, glass, painting, printmaking, sculpture and film and media programs.

To facilitate research-based collaborative work, we considered place-based topics, allowing for various modes of research, which would generate connections with the local environment. Since students from diverse disciplinary backgrounds and with different skill-sets enroll in the course, we deliberately selected a neutral topic of study, a locally sourced stone, in order to encourage a shared experience of discovery. Taking its name from the creek that defines the northwestern arm of the city of Philadelphia, the Wissahickon schist stone-a metamorphic rock-is widely used in historical construction in the area and well-recognized for its distinct specks of shiny mica and multi-toned layers of gray, blue, brown, and black. We decided to work with this stone as a departure point for diverse lines of inquiry into physical, historical, cultural, and social domains.

\section{WHAT IS COLLABORATION ANYWAY?}

One important discovery in early iterations of the course was that nearly every class member had a different understanding of and expectations for collaboration. It became necessary to establish specifically what we meant when we used the term. We define 


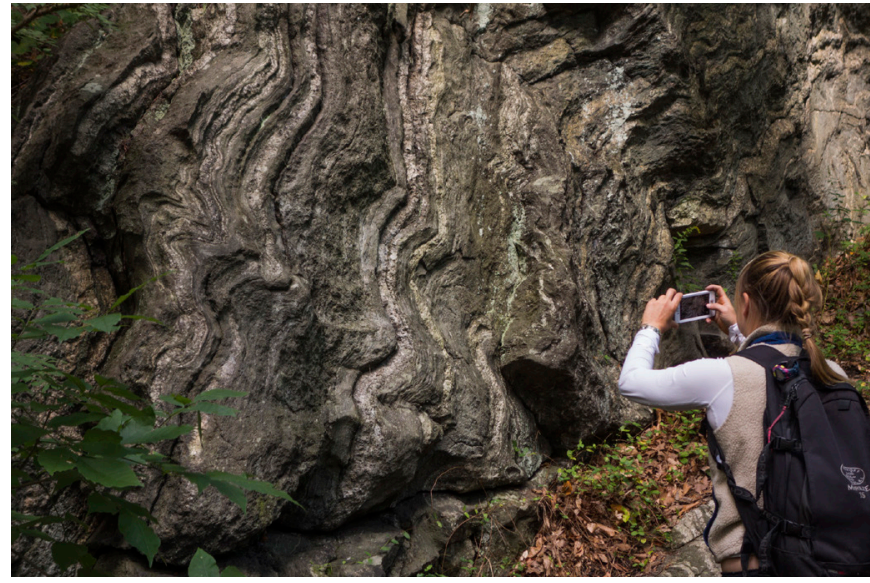

Figure 1. Student photographing Wissahickon schist during a group fiel work session in Wissahickon Valley Park, Philadelphia, Pennsylvania. (By Authors)

collaboration as a conscious and directed creative process that results in the amplification of ideas. Collaboration is distinct from partnerships, teamwork, or group work. Collaboration isn't simply working cooperatively toward a goal, but is instead about leveraging individual strengths to move beyond initial questions or existing boundaries. When functioning well, collaborations yield outcomes that could not or would not have been realized if one were working alone.

Beyond a shared understanding of the term collaboration, we also ascribe to the belief that awareness of o ne's process is essential for the continued success of that process, so we wanted to explicitly name and examine the properties and practices we recognized in our own past fruitful collaborations with the intention to make them prominent in the course. We described these behaviors as "Group Norms" 1 and included them among our course objectives as ways to articulate and nurture their development. Although there were many group norms we aimed to establish, the four most essential were; 1 ) a shared sense of commitment to the collaboration itself, independent of concerns for specific group production ${ }^{2}$ 3; 2) fair and equal access to shared knowledge, modes of communication, and modes of expression; 3) a habit of resilience when facing obstacles and barriers, and 4) an ability to improvise as needed with the understanding that things rarely unfold as expected or planned. These four stood out as universal and enduring, whereas depending on the dynamics of a collaboration others may be included and change over time. For this reason, with students, we reiterated the need for the collaborators to clearly establish their own group's norms, consistently pay attention to and periodically re-evaluate them. As collaborators in teaching, our pedagogical approach was to model the universal norms for healthy collaborations stated above in how we structured and ran the course: with deliberate time allotted for exploring our individual and common goals, ensuring equal access to tools and information, and a mode of working that permitted flexibility as well as improvisation.

\section{THE TYPICAL CREATIVE PROCESS}

Within the creative fields, the model for most student projectseither individual or collaborative-typically entails the use of a prompt, activities related to the investigation and response to the prompt, and a final outcome that is used as the basis for discussion and evaluation. E arly iterations of this course largely followed that traditional model, with some adjustments to how we conceived the types of activities during the middle exploratory phase. Class activities consisted of site visits with experts in geology, landscape architecture, and history to parts of the Wissahickon Creek valley and environs, fieldwork including surveys and documentation, research at local and regional archives, with a primary focus on collaborative work, complemented by some individual exercises (Figure 1). Not all activities were planned in advance, in an effort to leave room in the schedule for flexibility and impromptu exercises and discussions. What emerged for us as a natural and necessary approach is in fact reinforced as a strategy for healthy collaborations in psychologists Keith R. Sawyer and Stacy DeZutter's work. ${ }^{4}$ Sawyer and DeZutter put forth a provocative idea: the collaborative mode is not a direct outcome of carefully calibrated and scripted engagements, but an emergent property of social encounters in which participants' interactions are contingent upon moment-to-moment dynamics. In short, creative collaborations cannot be fully planned in advance, but the initial variables and conditions framing them can be thoughtfully prepared. With this insight in mind, we handled the course schedule as a "live" document and reserved space in daily p lanning, particularly toward the end of the semester when we anticipated an increase in momentum for each student collaboration.

Still operating within the prompt-activity-outcome paradigm, we defaulted to focusing on the qualities of the final presentation as the primary metric for the entirety of the semester. However, we found that examining a c ulminating o bject largely ignored what was really taking place within the group and how the group was functioning during the exploratory activities. Moreover, evaluating objects as outcomes was problematic as the course and project objectives were meant to establish particular group norms which needed their own assessment methods.

Our response to these observations is readily apparent in the way we iterated on the project briefs that students received to more closely align with the objectives and ethos of the course. Namely, we revised the structure and language of the text to reflect a "flip" in the hierarchy of ideas. When comparing two versions of the project briefs, one from the first time we taught the class to a nother from the final iteration, the titles are telling of the shift in our emphasis. The project we initially referred to as The Spatial and Sensory Survey, foregrounding the final product-the survey, evolved to Collaboration as Amplification, clearly indicative of the priority we placed on the process and underlying purpose of collaboration. Additionally, while the first document is structured such that the prompt comprises the top and visually more prominent section of the text, in the later versions we embed the prompt within a larger discussion about the process of collaboration. In 
effect, the reversal in positioning within the text mirrors a flip in our pedagogical priorities, where the process takes the foreground and its outcome recedes to the back, to a more subservient role. Furthermore, nuances in language describing the outcome suggest a move away from a prescriptive approach on our part to what may be characterized as a generative one. Initially, students find clear steps and parameters we outlined with suggested formats. In later versions of the prompts, we ask the groups to make many of these determinations based on how their processes unfold, also marking a transfer in responsibility from teacher to student.

\section{AN ALTERNATIVE MODEL IN CREATIVE COLLABORATIONS}

The evolution in our thinking in parallel with the project prompts led us to an alternative model in creative collaborations. We adopted an approach that de-emphasized the assessment of group output and instead focused primarily on ways to probe the question of how we work. This metacognitive approach required continuous reflection operating at the levels of the self and the collective. The complexities of group behaviors and dynamics sometimes required methods of assessment that ranged from traditional self reflections to more improvisational or unconventional group assessments.

\section{SELF REFLECTION}

In order to facilitate and record self-reflection, we asked students to complete a questionnaire prompting them to evaluate their working process within the collaboration. Different approaches we tried over the three iterations of the course experimented with quantitative and qualitative modalities. While we recognized the limits of quantitative assessments for such complex interpersonal dynamics, the exercise of reflecting on how the collaboration went and assigning a numeric value to certain aspects such as communication, mutual trust, and decision-making more readily revealed to us differences in perception among the individuals in the same collaboration. We used one such instance of misalignment in opinion

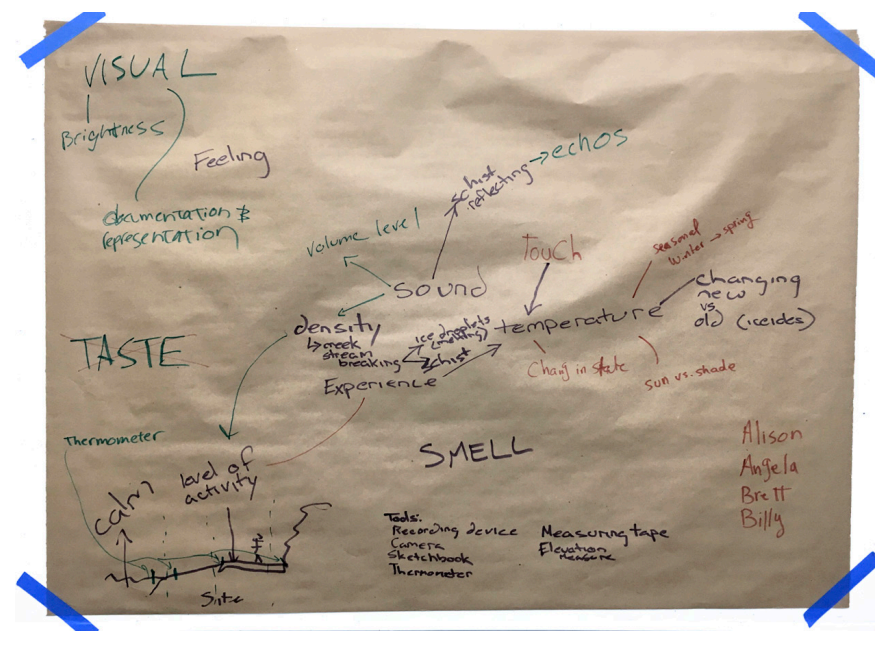

Figure 2. Selectio from a group mindmapping activit orchestrated to collecti ely investig te a topic or idea. (By Authors) to discuss with the entire class: one student described their collaborative dynamic as harmonious while another within the same group expressed sentiments of exclusion and tension. The ensuing discussion raised awareness toward the subjective nature of collaborative experience and reinforced the necessity to stay attuned to and address as needed the individuals' perspectives in collaborations.

In qualitative parts of the questionnaire, we took the opportunity to expand on the domain of a typical course evaluation and posed ideas that may not commonly be seen in the foreground of the creative process in arts/design/ architecture education. Some of these questions asked the students to reflect on the notion of group norms by identifying what they were and how and by whom they were established and describe their effect on the group's working process. Another question asked the students to reflect on a very specific issue such as the size of the collaboration-whether working in groups of two, three or five-and how it has influenced the collaborative dynamic. Yet another question prompted to students broaden their perspective on their creative process and consider a four-week period of collaboration in terms of the different stages the group passed, such as exploratory thinking, testing, archival research, etc. And another question with a broad outlook returned to the core principle of what makes group work a true collaboration and asked the students to elaborate on whether any of the group's ideas, processes, or methods were amplified by the collaboration.

\section{COLLECTIVE REFLECTION}

At the level of the collective, we devised a series of exercises to facilitate different modes of reflection, many of which were developed in response to behaviors we observed within the group. The common thread running through all of these analytics is that they reveal how we behave in collaborations-how we work-through an external, metacognitive lens. From different angles, student collaborators step away from the work itself to gain the vantage point of how they actually operate within the group in comparison to how they thought they did.

In one such exercise, as instructors, we took on the metacognitive role in order to exhibit relationships that were not readily self-evident to the students. We implemented a "mindmapping" tool for visually collecting and organizing the ideas group m embers generated in real-time (Figure 2). By including every individual's input on the map and not editing out any ideas but collectively organizing them relative to o ne a nother, we were able to treat the visual record as a "host" for diverse but interrelated voices, in conversation. We found this type of mapping tool most appropriate when dealing with a prompt that benefited from expansive and non-linear thinking, such that students could see more clearly the results of collective exploration in what 


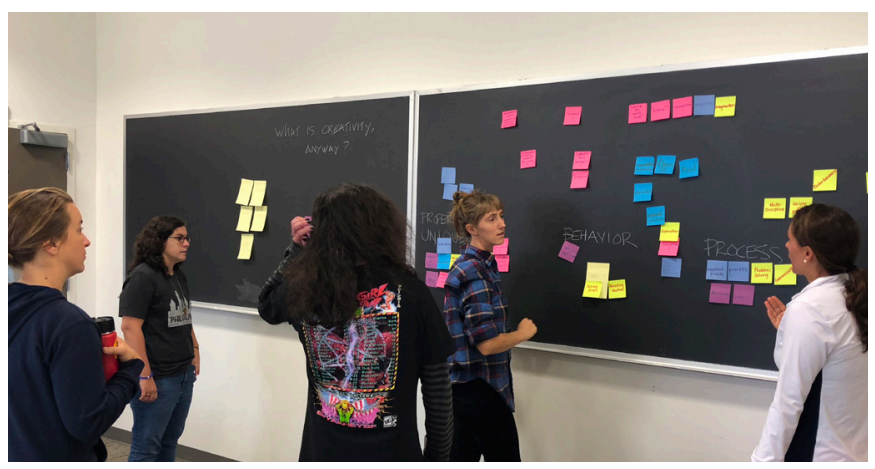

Figure 3. Communicati $\mathrm{n}$ cards and Post-it notes used in a group discussion and analysis in response to the questio "What is Creativit anyway?" (By Authors)

otherwise may seem as "inefficient" and meandering work sessions.

For another type of collective reflection, we relied on communication cards or Post-it notes, simple mental records that offered an opportunity to generate thoughts and ideas which were then collectively discussed, analyzed and organized. The communication cards provided a way for more reserved individuals to more fully engage and participate from the very outset of a g roup activity.

One such exercise of collective reflection addressed the question "What is creativity, anyway?" Within a matter of minutes of outlining the prompt, each student generated at least a h alf dozen different responses in the form of a word or a phrase. The results were discussed and more deeply investigated with instructors acting as moderators. The resulting conversation and visual map revealed that the word "creativity" had multiple meanings that could be mapped across domains that included behaviors, process-es, properties, popular myths, and more (Figure 3).

The purpose of analyzing this specific question about creativity was to prompt a deeper investigation into a term that is so often

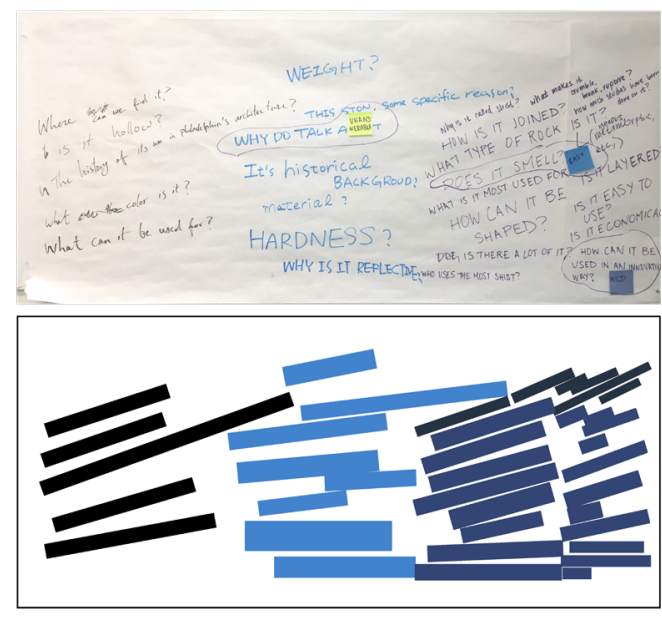

Figure 4. A visual analysis of a brainstorming activit that compares the relati e interactio between two di erent groups. Further analysis revealed that the more interacti e group yielded more linked and unique ideas. (By Authors) used as a general placeholder for more specific meanings and to have students who will be working collaboratively to have a greater shared understanding for its complexities, but also to demystify a critical aspect of working collaboratively. If creativity is collectively understood as sometimes being a process (as opposed to a magical attribute or individual characteristic) then it is more likely that an individual might see themselves as potentially participating and contributing to that creative process. In this particular context, the collective reflection p rovided an o pportunity for individuals to see for themselves that they might have a role to play in a creative, collaborative enterprise.

Another traditional exercise with an unconventional outcome was a brainstorming session intended to have students practice exploring a particular topic while interacting and building upon one another's ideas. Generative thinking and linking and building upon other's ideas were cited as the primary goal of the exercise. After a discussion about the methods and purposes for brainstorming, each student was given a different colored marker and they worked together for ten minutes in groups of four, recording their ideas on a large sheet of paper.

During the session, we observed that some groups engaged in more discussion and interaction than others. Then, instead of analyzing the content of the generative exercise, we opted to a nalyze the remnants of the activity itself to try to quantify the relationship between group interaction and the number of unique or linked ideas. We converted the text for each word or phrase into a solid block of color that corresponded to each pen color. Then, we counted the number of distinct ideas and the number of links between each idea (Figure 4).

In this example, the mix of colors represents the relative exchange and interplay of ideas and the degree of conversation among the group. With a simplified visual analysis of the group's working process, a relative difference of interaction becomes more evident. The group with the ideas arranged in discrete columns had

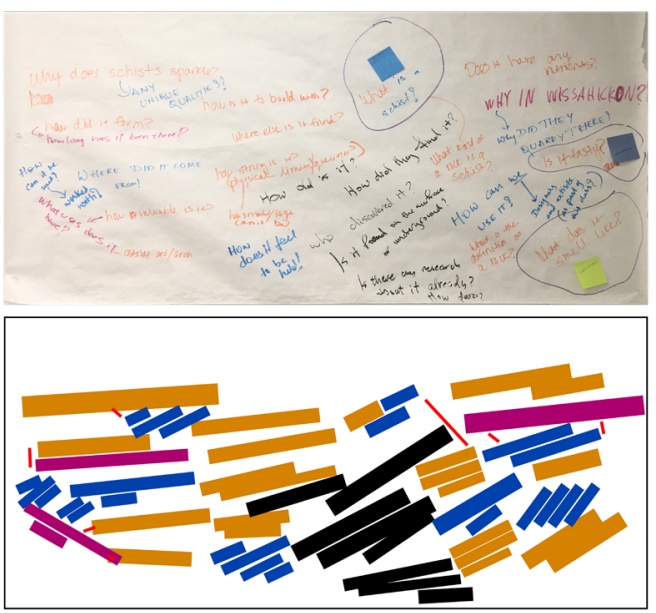




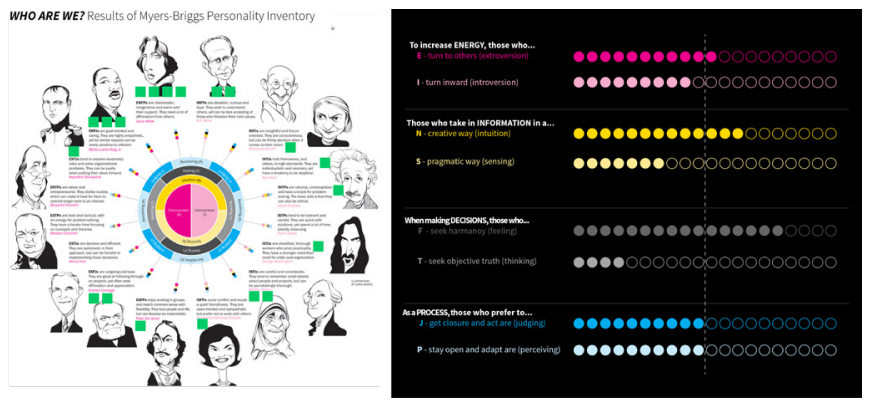

Figure 5. Who Are We? A visual overview and analysis of Myers-Briggs Personality inventory results for students and faculty in the course. (By Authors)

$30 \%$ fewer unique ideas and almost $80 \%$ fewer linked ideas than the group with the text that is nearly woven together. Of course, this quantitative analysis isn't statistically significant in any way, and it doesn't evaluate the quality of ideas generated, but since the primary objective for this exercise was to generate a high volume of ideas, the method of assessment needed to a lign with that goal.

This visual analysis of group dynamics was the result of a planned activity n ot unfolding q uite as expected. Even though the task itself was quite simple, and most participants thought they engaged with the activity in ways that were sufficient, seeing a visual analysis of interpersonal communication was revealing to nearly all and highlighted for many that their own impressions for how they were working were quite different from what an external analysis revealed.

The question of how we work is inextricably tied to who we are-an awareness that is essential to navigating dynamics of group work but is rarely discussed at w ork. We f ound that assessment of personality traits codified the composition of the collaborators and
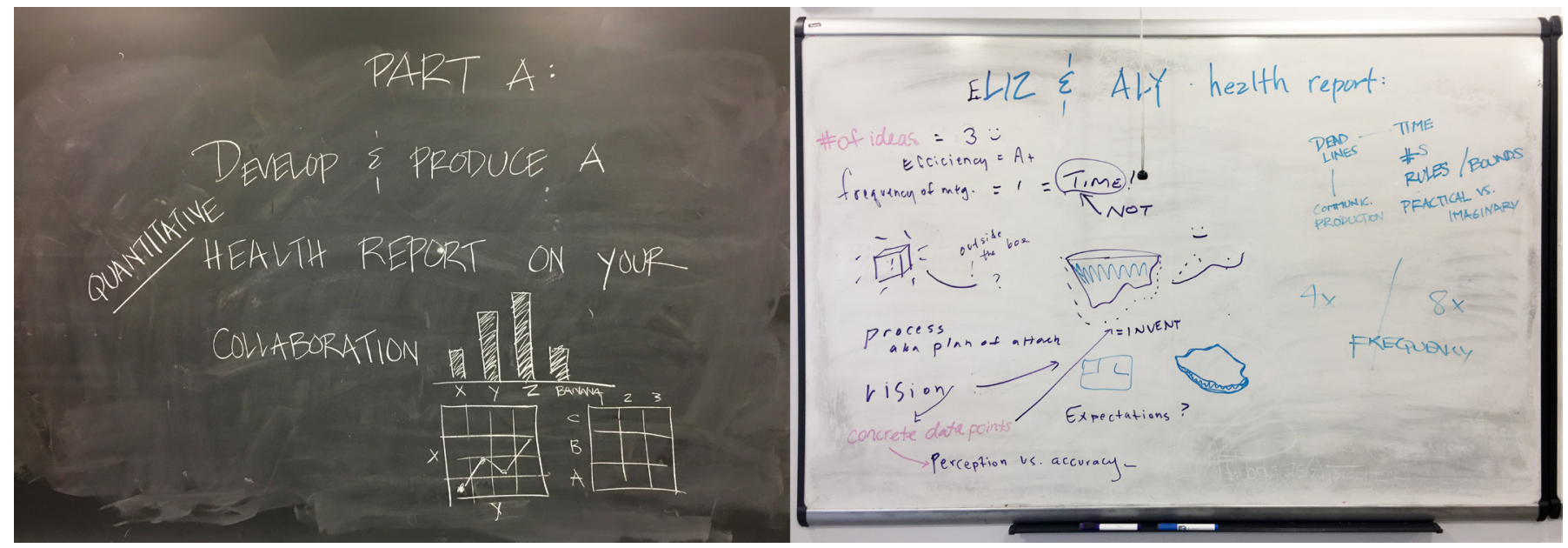

Figure 6. The prompt and results from a collaborati e group asked to collecti ely discuss, quantif, and diagram the health of the collaboratio. (By Authors) 
their roles as participants and to take some time to be observers of behavior to consider any issues or emerging group norms that were keeping the group from a mplifying ideas and realizing potential.

\section{HOW DO WE WORK? NEW PERSPECTIVES}

Working collaboratively and teaching methods of collaboration is at turns exhilarating and unnerving as the challenges, uncertainties, and insecurities of creating while working alone not only remain with e ach individual but are perhaps even a mplified when working within a collective. Reorienting this course towards metacognitive attentiveness and away from being primarily concerned with final output has provided some new perspectives for us as teachers. Most notably, focusing on Process-and treating it with an attentive and analytical eye-simply feels different at nearly every stage of the course, certainly for the instructors, and possibly for the students as well.

We've learned that what works with o ne group of s tudents may not work in the next. Personalities and group dynamics do matter and they change from one group to another, even within the same group over time. As instructors, we've been called upon to be more improvisational in our teaching and to rely less upon "scripted engagements." While we still work within a structure and a plan, we've become more comfortable in making adjustments within the moment to more readily address what any given moment may require. In this way, o ur teaching has possibly developed an e mergent property sometimes seen within a h ealthy collaboration.

The essential group norm of "a shared sense of commitment to the collaboration itself, independent of concerns for specific group production" has been a real challenge for us to consistently initiate and nurture. Each student comes to the course with different ambitions, goals and expectations. When students work alone, there's little mixing of these variables with classmates, and the consequences for a lack of commitment are isolated. Within a classroom collaboration, of course, everyone's varying levels of commitment are co-mingled, and we've found the group itself probably exercises as much or more influence on behaviors and norms than we might as instructors. Nonetheless, students often look to the instructors to somehow instill a s imilar shared sense of commitment in every student. This challenge will certainly endure in future course iterations.

Assessment, especially quantitative assessment of individual student performance within a collaboration, is extraordinarily difficult, if not impossible. It is clear that consistently practicing different modes of reflection to collectively step into the metacognitive viewpoint is essential and may even be a necessary substitute for typical assessment. To that end, we intend to continue to devise methods that combine reflective quantitative and qualitative modalities for individual and group student feedback and learning. Whether merely perceived or real, o ur sensitivity a nd concern about a lack of individual assessment within the course is perhaps a reflection of institutional and cultural expectations exclusively o riented toward individual performance and outcomes.

Similar to the ambiguity of assessment, another new perspective in our approach has meant that "Success" in a collaboration may be ambiguous or difficult for goal-oriented individuals to recognize or value in the moment. In the context of an Art and Architecture School, attention toward "soft skills" such as Process seems essential, but the value of such experiences isn't always self-evident in the moment or even at the conclusion of a c ourse.

In advance of the next iteration of teaching this course, we have found the exercise of formalizing our thoughts for a larger audience via this conference has created yet another level of metacognition as far as how we, as educators in collaboration, work as well. B oth our experience teaching the course and our reflections through writing about it have clarified for us that teaching collaboration across creative disciplines requires re-education, on the part of the students as well as the teachers.

\section{Notes}

1. A more in-depth discussion on work group dynamics, specifically at Google, can be found here: https://www. nytimes.com/2016/02/28/magazine/what-google-learnedfrom-its-quest-to-build-the-perfect-team.html

2. a shared sense of commitment has sometimes proven difficult to facilitate and ensure at the individual level. Instead, it has largely appeared as an emergent property from other group norms.

3. This is also supported by research conducted by experimental psychologists P.B. Carr and G.M. Walton, who found that, in true collaborations, individuals must trust in each other's commitment to the joint endeavor while maintaining a certain level of autonomy.

4. Sawyer, DeZutter, "Distributed Creativity: How Collective Creations Emerge from Collaboration," Psychology of Aesthetics, Creativity, and the Arts, 2009, Vol. 3, No. , 81-92.

5. More information on the work done at MyersBriggs foundation can be found here: https://www. myersbriggs.org/my-mbti-personality-type/mbti-basics/ home.htm?bhcp=1 